\title{
Spin-orbit-coupled quantum wires and Majorana fermions on zigzag edges of monolayer transition-metal dichalcogenides
}

\author{
Rui-Lin Chu, ${ }^{1}$ Gui-Bin Liu, ${ }^{2,3}$ Wang Yao, ${ }^{3}$ Xiaodong Xu, ${ }^{4,5}$ Di Xiao, ${ }^{6}$ and Chuanwei Zhang ${ }^{1, *}$ \\ ${ }^{1}$ Department of Physics, University of Texas at Dallas, Richardson, Texas 75080, USA \\ ${ }^{2}$ School of Physics, Beijing Institute of Technology, Beijing 100081, China \\ ${ }^{3}$ Department of Physics and Center of Theoretical and Computational Physics, The University of Hong Kong, Hong Kong, China \\ ${ }^{4}$ Department of Physics, University of Washington, Seattle, Washington, USA \\ ${ }^{5}$ Department of Material Science and Engineering, University of Washington, Seattle, Washington, USA \\ ${ }^{6}$ Department of Physics, Carnegie Mellon University, Pittsburgh, Pennsylvania 15213, USA \\ (Received 7 October 2013; revised manuscript received 31 March 2014; published 22 April 2014)
}

\begin{abstract}
Majorana fermions, quantum particles with non-Abelian exchange statistics, are not only of fundamental importance, but also building blocks for fault-tolerant quantum computation. Although certain experimental breakthroughs for observing Majorana fermions have been made recently, their conclusive detection is still challenging due to the lack of proper material properties of the underlined experimental systems. Here we propose a platform for Majorana fermions based on edge states of certain nontopological two-dimensional semiconductors with strong spin-orbit coupling, such as monolayer group-VI transition-metal dichalcogenides (TMDs). Using first-principles calculations and tight-binding modeling, we show that zigzag edges of monolayer TMD can host a well isolated single edge band with strong spin-orbit-coupling energy. Combining with proximity induced $s$-wave superconductivity and in-plane magnetic fields, the zigzag edge supports robust topological Majorana bound states at the edge ends, although the two-dimensional bulk itself is nontopological.
\end{abstract}

DOI: 10.1103/PhysRevB.89.155317

PACS number(s): 74.45.+c, 75.70.Tj, 73.20.-r

\section{INTRODUCTION}

Majorana fermions [1] are quantum particles that are their own antiparticles, and they satisfy non-Abelian exchange statistics. The latter is the key for their potential use in faulttolerant topological quantum computation [2], which makes their experimental realization an extremely important task from the long-term technological perspective. In the past two decades, some exotic condensed matter/cold atom systems [29] have been proposed to support Majorana fermions. The experimental breakthrough occurs only recently [10-13], using heterostructures consisting of conventional $s$-wave superconductors and semiconducting nanowires subjected to an external magnetic field, where certain signatures of Majorana fermions were observed. However, there are a few material complications inherent to semiconductor nanowires that may prevent the experimental signature from being conclusive [14-19]: (i) The large diameters of the nanowires yield multiple occupied transversal sub bands, resulting in complications for the superconductor proximity effect and the chemical potential level [20-22]; (ii) the spin-orbit coupling in these wires is rather weak, which renders the Majorana physics extremely vulnerable to disorder, making it challenging to exclude alternative interpretations of the experimental signature based on disorder effect [14,23,24]; (iii) the random growth process of nanowires also makes it hard to build a nanowire network [25] to detect the statistics of Majorana fermions.

Amid the above difficulty, it is critically important to look for other 1D conducting states to realize Majorana fermions. A natural way is to consider $1 \mathrm{D}$ edge states of a $2 \mathrm{D}$ material. In this context, helical edge states of 2D quantum spin Hall

\footnotetext{
*chuanwei.zhang@utdallas.edu
}

insulators (QSHIs) have been proposed to support Majorana fermions [26-28]. However, so far the QSHIs have only been realized in semiconductor heterostructures and are subjected to stringent growth conditions. Furthermore, the bulk itself of a QSHI is generally not a good insulator because of the relatively small band gap (about $10 \mathrm{meV}$ ). It is therefore natural to ask whether the edge states of nontopological 2D materials with a large bulk band gap can support Majorana fermions. While in pursuit of such platforms a few key material properties are of particular interest: (i) The compounds must have heavy elements that can generate strong spin-orbit coupling (SOC) necessary for robust 1D topological superconductors [29,30]; and (ii) 2D atomically thin materials with honeycomblike lattice structures (i.e., similar as graphene), which are more likely to support single band edge states.

In this paper we demonstrate this idea by showing that $1 \mathrm{D}$ zigzag edges of a class of $2 \mathrm{D}$ semiconductors, monolayer group-VI transition-metal dichalcogenides (TMDs), provide a promising platform for studying 1D topological superconductors with a single transversal band and strong SOC energy. Using both first-principles calculations and tightbinding modeling, we show that the chalcogen-terminated zigzag edges of these 2D semiconductors support edge bands with strong Rashba-type SOC and are well separated from the bulk bands. By utilizing a minimal realistic tight-binding model, we numerically confirm the existence of zero-energy Majorana states at the two ends of the edge in the presence of proximity induced $s$-wave superconductivity, and their robustness against disorders. The proposed system has identical topological properties with the well-established nanowire systems [31]. Our findings point out a pathway for searching for Majorana fermions using edge states of widely existing 2D nontopological semiconductors. 
(a)

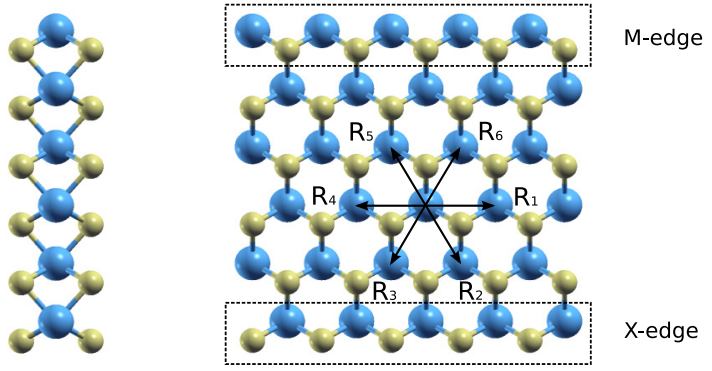

(b)

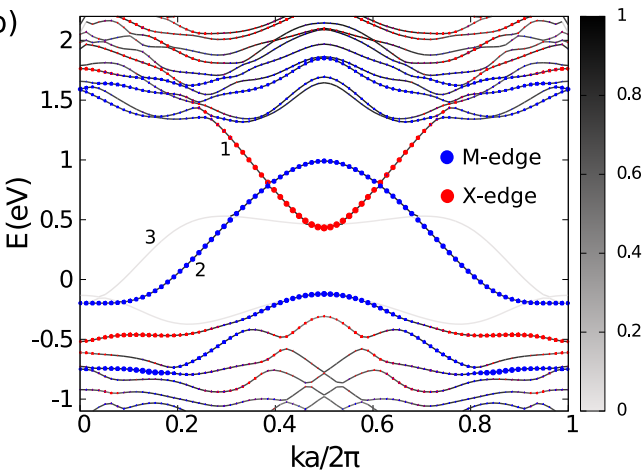

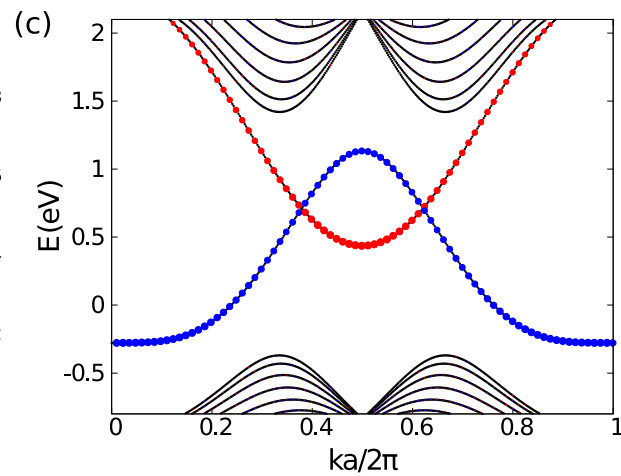

FIG. 1. (Color online) Isolated single edge band in monolayer $\mathrm{MX}_{2}$. (a) Side and top view of a monolayer $\mathrm{MX}_{2}$ zigzag ribbon. $R_{i}$ are the vectors connecting the nearest $\mathrm{M}$ atoms. The ribbon is infinite in the $R_{1}$ and $R_{4}$ directions. The lower and upper edges of the ribbon are referred to as the $\mathrm{X}$ edge and $\mathrm{M}$ edge, respectively. The ribbon's width is measured by the number of zigzag chains $N_{c}$ in width. (b) DFT band structure of a $\mathrm{WSe}_{2}$ zigzag ribbon with $N_{c}=8$, not including SOC. The grayscale bar represents the total orbital $\left(d_{z^{2}}+d_{x y}+d_{x^{2}-y^{2}}\right)$ weight of the band. The color dots represent the orbital $\left(d_{z^{2}}+d_{x y}+d_{x^{2}-y^{2}}\right)$ weight (with larger dot for larger weight) from the $\mathrm{M}$ atoms on the $\mathrm{X}$ or $\mathrm{M}$ edge. Three pairs (marked 1 to 3 ) of well localized in-gap edge bands can be identified, one (red dot) is on the X edge and the other two (blue dot and light gray) are on the M edge. The two inequivalent valleys $K$ and $K^{\prime}$ are located at $k a / 2 \pi=1 / 3$ and $2 / 3$, respectively. $a=3.31 \AA$ is the bulk's lattice constant. Fermi surface is at $E=0$. Plots for $\mathrm{MoS}_{2}, \mathrm{MoSe}_{2}$, and $\mathrm{WS}_{2}$ have similar patterns. (c) Same as (b) with tight-binding model and $N_{c}=20$.

\section{TIGHT-BINDING MODELING}

We consider four different TMDs: $\mathrm{MoS}_{2}, \mathrm{MoSe}_{2}, \mathrm{WS}_{2}$, and $\mathrm{WSe}_{2}$, but will present our results in the following mainly using $\mathrm{WSe}_{2}$ as a representative. Monolayer TMDs are atomically thin 2D direct-band-gap semiconductors with exotic coupled spin and valley physics [32] and excellent optical properties, as demonstrated in recent experiments [33-39]. Structurally monolayer $\mathrm{MX}_{2}$ is a tri-layer X-M-X sandwich. Within each layer, $\mathrm{M}$ and $\mathrm{X}$ atoms form $2 \mathrm{D}$ hexagonal lattices. When viewed from the top it shows a honeycomb structure. The 2D bulk of monolayer $\mathrm{MX}_{2}$ has a direct band gap of $1.5-2 \mathrm{eV}$ located at the corners of its 2D hexagonal Brillouin zone called valleys, which has been confirmed by both first-principle studies and experiments [32-39]. The bulk's edges can be classified as zigzag and armchair types like in graphene. Due to the lack of inversion symmetry in the monolayer, the zigzag edges can be further classified as X-terminated and M-terminated, which correspond to the lower and upper edges of the ribbon shown in Fig. 1(a). We refer to them as $\mathrm{X}$-edge and M-edge, respectively. It is already known from STM measurements that the zigzag edges of triangular shaped monolayer $\mathrm{MoS}_{2}$ nanoflakes support multiple pairs of 1D metallic edge states [40]. The edges of these nanoflakes are later identified as Mo-edge with passivated $\mathrm{S}$ atoms [41]. For a zigzag $\mathrm{MX}_{2}$ ribbon shown in Fig. 1(a), the edge states exist on both the $\mathrm{M}$ edge and $\mathrm{X}$ edge.
In addition to the density functional theory (DFT) calculations, insight into the underlying physics can be obtained from a minimal tight-binding model that is constructed by considering the lattice symmetry and the corresponding crystal field splitting. It is known that the valence band maximum and conduction band minimum of monolayer $\mathrm{MX}_{2}$ consist mainly of $\mathrm{M}$ atom's $d$ orbitals. Thus to describe the low energy band structure of the monolayer's bulk, it is sufficient to consider the $d$ orbitals from the M atoms [32]. The trigonal prismatic coordination of the $\mathrm{M}$ atom splits its $d$ orbitals into three groups: $A_{1}^{\prime}\left(d_{z^{2}}\right), E^{\prime}\left(d_{x y}, d_{x^{2}-y^{2}}\right)$, and $E^{\prime \prime}\left(d_{x z}, d_{y z}\right)$. The monolayer's mirror symmetry in the $\hat{z}$ direction permits hybridization only between the $A_{1}^{\prime}$ and $E^{\prime}$ groups. This allows us to consider three orbitals of $d_{z^{2}}, d_{x y}$, and $d_{x^{2}-y^{2}}$ for a minimal tight-binding model. We refer the readers to Ref. [42] for detailed descriptions of this tight-binding model including the symmetry analysis and material specific parameters fitted from first-principle calculations. The tight-binding model is able to capture the essential physics of the monolayer, including the direct band gaps at the $K$ and $K^{\prime}$ valleys, the degeneracy of the band edges and the valley contrast spin splitting of the valence band due to SOC, etc. [32,42].

In simple languages this tight-binding model only considers $\mathrm{M}$ atoms' on-site energies and electron hopping along the six vectors connecting the nearest $\mathrm{M}$ atoms (marked as $R_{1} \sim R_{6}$ in Fig. 1(a). Without considering SOC and the spin degree of freedom, the tight-binding Hamiltonian can be written as a 

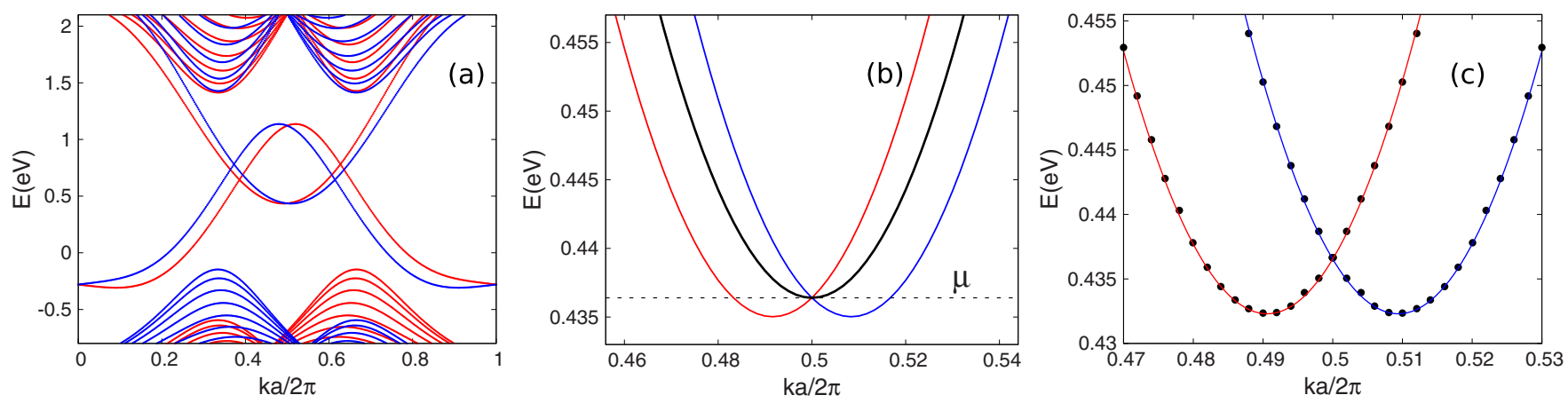

FIG. 2. (Color online) X-edge band minimum. (a) Tight-binding band structure for zigzag nanoribbon with SOC turned on. The color marks spin direction with red $=$ spin up, blue $=$ spin down. $N_{c}=20, \lambda=460 \mathrm{meV}$. (b) Zoom in of the X-edge band minimum of (a) at $k a=\pi$. The black line is without SOC. The dashed line marks the chemical potential used in the BdG calculation in Fig. 3. (c) Same as (b) for Fig. 1(b) with SOC turned on, where the dots are DFT results and the lines represents the best fit from Eq. (3).

$3 \times 3$ matrix

$$
\mathcal{H}(\mathbf{k})=\left(\begin{array}{lll}
H_{11}^{11} & H_{11}^{12} & H_{12}^{12} \\
& H_{11}^{22} & H_{12}^{22} \\
\text { c.c. } & & H_{22}^{22}
\end{array}\right),
$$

in the $\mathbf{k}$ space. Here the basis is taken as $\left\{\left|\phi_{1}^{1}\right\rangle=d_{z^{2}},\left|\phi_{1}^{2}\right\rangle=\right.$ $\left.d_{x y},\left|\phi_{2}^{2}\right\rangle=d_{x^{2}-y^{2}}\right\}$, and $H_{i j}^{\alpha \beta}$ represents the matrix element between $\left|\phi_{i}^{\alpha}\right\rangle$ and $\left|\phi_{j}^{\beta}\right\rangle$, and is obtained from the Fourier transformation of the real space tunneling matrix between neighboring sites.

\section{RASHBA-TYPE SOC IN THE EDGE BAND}

We proceed to include SOC and demonstrate how the Rashba-type SOC in the edge band is generated. The $\mathbf{L} \cdot \mathbf{S}$ type $\mathrm{SOC}$ in $\mathrm{MX}_{2}$ originates from the $d$ orbitals of the heavy $\mathrm{M}$ atoms (Mo or W) $[32,43]$. In the monolayer's bulk, the spin-orbit term can be described as

$$
\mathcal{H}_{\mathrm{SO}}=\frac{\lambda}{2}\left(S_{x} \otimes L_{x}+S_{y} \otimes L_{y}+S_{z} \otimes L_{z}\right),
$$

where $S_{i}$ and $L_{i}$ represent the spin and orbital angular momentum operator, respectively. It turns out in the basis of $\left\{\left|\phi_{1}^{1}\right\rangle,\left|\phi_{1}^{2}\right\rangle,\left|\phi_{2}^{2}\right\rangle\right\}$ the $L_{x}$ and $L_{y}$ are both $0_{3 \times 3}$ matrices, which enables us to write the total Hamiltonian in a spin-decoupled form

$$
\begin{aligned}
\mathcal{H}^{\prime}(\mathbf{k}) & =I_{2} \otimes \mathcal{H}(\mathbf{k})+\mathcal{H}_{\mathrm{SO}} \\
& =\left(\begin{array}{cc}
\mathcal{H}(\mathbf{k})+\frac{\lambda}{4} L_{z} & 0 \\
0 & \mathcal{H}(\mathbf{k})-\frac{\lambda}{4} L_{z}
\end{array}\right),
\end{aligned}
$$

where

$$
L_{z}=\left(\begin{array}{ccc}
0 & 0 & 0 \\
0 & 0 & 2 i \\
0 & -2 i & 0
\end{array}\right) .
$$

Here the upper and lower sub-blocks represent the spin up and down, respectively. It is noted that both $\mathcal{H}(\mathbf{k})$ and the total Hamiltonian $\mathcal{H}^{\prime}(\mathbf{k})$ are time-reversal invariant. The SOC term $\mathcal{H}_{\text {so }}$ leads to large valley contrast spin splitting of the valence band, which has been observed in experiments [33,35-39].
In Figs. 1(b), 1(c), and 2 we present both the DFT and tight-binding band structure of the $\mathrm{WSe}_{2}$ zigzag ribbon, where the edge states localized on different edges are marked correspondingly. The width of the ribbon is chosen to be within the experimental range (i.e., 1-3 nm) [44-46]. Because of the narrow nanoribbon, the two-valley band structure in the bulk is not developed. However, the valleys are not relevant in the proposed edge physics below. The electrons from these edge bands dominantly resides on the $\mathrm{M}$ atoms of the zigzag edges. Comparing with the DFT band structure, we see the tight-binding model can successfully capture the parabolic edge bands on both the $\mathrm{X}$ and $\mathrm{M}$ edge. It's worth mentioning that the effective SOC we find in the parabolic $\mathrm{M}$-edge band is generally larger than in the $\mathrm{X}$-edge band. Nevertheless the $\mathrm{X}$-edge is preferable for two main reasons. First, the $\mathrm{X}$ edge hosts a single edge band while the $\mathrm{M}$ edge hosts multiple edge bands; second, the $\mathrm{X}$ edge is structurally very stable while the $\mathrm{M}$ edge can be dramatically affected by edge passivations [44,47-49]. Hereafter, we will focus on the $\mathrm{X}$-edge band.

Note that the existence of such edge bands originates from the broken lattice symmetry at the edge and is not determined from the bulk topology of monolayer TMDs like that in topological insulators (i.e., by bulk-boundary correspondence). In fact, the bulk of monolayer TMDs is nontopological. As a result, the edge state is well localized on a 7 single atom chain, and the finite size effect that causes the hybridization between two edges in topological insulators is negligible for the nanoribbons considered here.

In Fig. 2(b) we compare the X-edge band before and after turning on the SOC. Apparently the $\mathcal{H}_{\text {SO }}$ can be viewed as a TRS breaking perturbation term, whose effect is slightly shifting the spin up branch to the left and spin down branch to the right. The whole band structure nevertheless remains symmetric about $k a=\pi$ because of the TRS. Accordingly the low energy effective 1D Hamiltonian for the X-edge band can be written as

$$
\mathcal{H}_{\text {eff }}\left(k^{\prime}\right)=\frac{1}{2 m^{*}} k^{\prime 2}+\alpha_{R} k^{\prime} \sigma^{z}+C,
$$

where $\sigma^{z}$ is the $z$ component of the Pauli matrix, $k^{\prime}=k-$ $\pi / a$, and $C$ is a constant. Up to a unitary transformation, 
TABLE I. Effective mass and Rashba velocity for TMDs' S(Se)-edge band and semiconductor nanowires. SOC energy is defined as $E_{\mathrm{SO}}=\frac{1}{2} m^{*} \alpha_{R}^{2}$.

\begin{tabular}{lcccccc}
\hline \hline & $\mathrm{MoS}_{2}$ & $\mathrm{MoSe}_{2}$ & $\mathrm{WS}_{2}$ & WSe $_{2}$ & InAs (Ref. [8]) & InSb (Ref. [10]) \\
\hline$m^{*}\left(m_{e}\right)$ & 0.28 & 0.24 & 0.33 & 0.31 & 0.04 & 0.015 \\
$\alpha_{R}(\mathrm{eV} \mathrm{\AA})$ & 0.12 & 0.11 & 0.33 & 0.46 & 0.06 & 0.2 \\
$E_{\text {SO }}(\mathrm{meV})$ & 0.26 & 0.2 & 2.3 & 4.3 & 0.01 & 0.05 \\
\hline \hline
\end{tabular}

this Hamiltonian is equivalent to that for the semiconductor nanowires with Rashba-type SOC [6,10-12,20-22,25]. Here $\alpha_{R}$ is the effective Rashba velocity. In Table I the effective mass and $\alpha_{R}$ fitted from our first-principle calculations for $\mathrm{MoS}_{2}, \mathrm{MoSe}_{2}, \mathrm{WS}_{2}$, and $\mathrm{WSe}_{2}$ are listed. These parameters are orders of magnitude larger than their semiconductor nanowire counterparts, especially for $\mathrm{WS}_{2}$ and $\mathrm{WSe}_{2}$.

\section{MAJORANA END STATES}

To create a 1D topological superconductor, we introduce superconducting pairing through proximity effects by depositing the $\mathrm{MX}_{2}$ monolayer on top of a conventional $s$-wave superconductor ( $\mathrm{Nb}, \mathrm{NbSe}_{2}$, etc.), as illustrated in Fig. 3(a). The $\mathrm{MX}_{2}$ monolayers have X-M-X layer thickness $\approx 3 \AA$, which is well within the superconducting coherence length of a typical $s$-wave superconductor. The selected monolayer can either be (1) a zigzag nanoribbon or (2) a large monolayer sample with an identified $\mathrm{X}$ edge. A top gate can then be applied locally to tune the chemical potential. We note that in the first case there are coexisting edge states on the $\mathrm{M}$ edge as shown in the ribbon's band structures [Figs. 1(b) and 1(c)]. These M-edge states are well localized on the M-edge's $\mathrm{M}$ atoms. To realize a topological superconducting state, the chemical potential $\mu$ need be tuned to be around the X-edge band bottom near $k=\pi / a$ [see Fig. 2(b)]. The corresponding M-edge states at this chemical potential occur at momenta far away from $k=\pi / a$. In that region, an even number of Moedge bands are cut at the Fermi surface, which do not affect the topological properties of the system [6]. Therefore the M-edge states do not interfere with the topological superconducting state on the $\mathrm{X}$ edge, which is also confirmed in our following numerical simulations. Such coexistence of edge bands is completely eliminated for the second case, where the $\mathrm{X}$ edge can be well isolated. For this case the gate is only required to cover the selected segment of the edge since the bulk maintains a large band gap.

To demonstrate the functionality of the proposed setup, we carry out a numerical simulation in $2 \mathrm{D}$ with the tightbinding model. We adopt the ribbon structure for this purpose

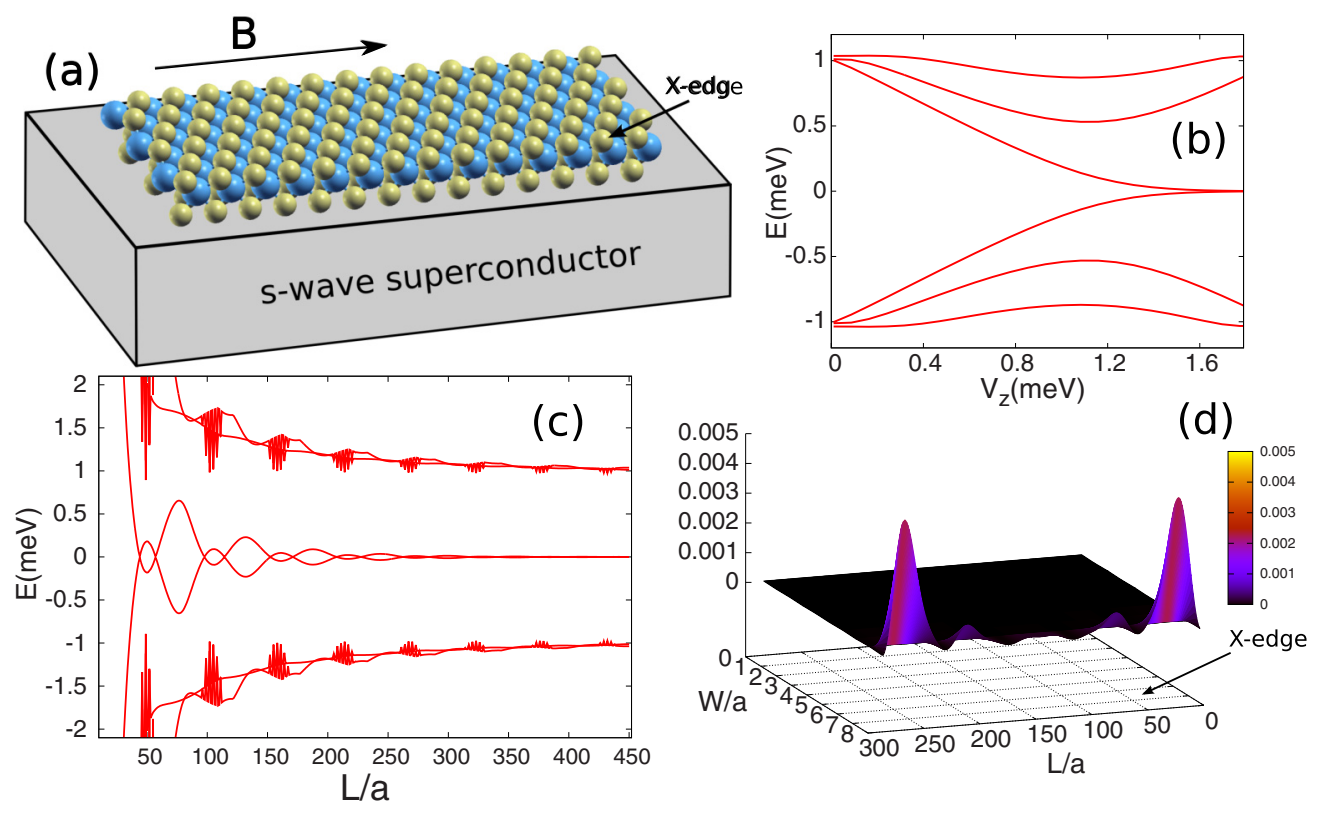

FIG. 3. (Color online) Majorana zero-energy mode. (a) A monolayer $\mathrm{MX}_{2}$ zigzag ribbon deposited on top of an $s$-wave superconductor. A top gate can be applied to tune the chemical potential. An external magnetic field is applied parallel to the zigzag edge in order to make the system a topological superconductor. (b) The emergence of the zero-energy mode. $L / a=400$. (c) Evolution of low energy spectrum with ribbon's length $L . V_{z}=2.0 \mathrm{meV}$. In (b) and (c) only six modes closest to zero energy are plotted. (d) Real-space distribution of the zero-energy mode over the ribbon for $L / a=300$. The $3 \mathrm{D}$ view angle is set to be the same as that in (a). Other parameters are $N_{c}=10, \Delta=1.0 \mathrm{meV}$, and $\mu=0.4364 \mathrm{eV}$. 
as illustrated in Fig. 3(a). Because of their excellent structural stability, TMDs zigzag nanoribbons can be synthesized with uniform width and smooth edges without defects [44-46]. To drive the system into a topological superconducting state, an in-plane magnetic field is applied to create a Zeeman splitting gap at the band crossing point.

The Zeeman term induced by the magnetic field reads

$$
\mathcal{H}_{Z}=V_{z} \sum_{i, l \alpha \beta} c_{i, l \alpha}^{\dagger} \sigma_{\alpha, \beta}^{x} c_{i, l \beta},
$$

where $c_{i, l \alpha}^{\dagger}$ is the creation operator for the electron on site $i$ with orbital index $l(1 \sim 3)$ and spin index $\alpha$ and $\beta$. We have assumed the magnetic field is in the $x$ direction, but nevertheless any in-plane magnetic field would work equivalently. The proximity effect induced superconducting paring term writes

$$
\mathcal{H}_{\mathrm{SC}}=\sum_{i, l}\left(\Delta c_{i, l \uparrow}^{\dagger} c_{i, l \downarrow}^{\dagger}+\text { H.c. }\right),
$$

where for simplicity we assume a uniform intraorbit pairing strength. Denoting the lattice version of the ribbon's Hamiltonian in Eq. (3) as $\mathcal{H}_{0}$, we then solve the corresponding BdG equation for the full Hamiltonian

$$
\mathcal{H}=\mathcal{H}_{0}-\mu+\mathcal{H}_{Z}+\mathcal{H}_{\mathrm{SC}}
$$

to get the low energy spectrum. The emergence of the zero-energy mode with increasing Zeeman splitting is shown in Fig. 3(b). In Fig. 3(c) we show the evolution of the six lowest energy modes with the ribbon's length. As a signature of Majorana fermions [50], the zero-energy modes show an oscillating energy splitting with an exponentially decaying envelope. When the ribbon is sufficiently long the zero-energy mode as well as the excitation gap become well defined. We notice that the fast alternating modes in the excitation gap of a short ribbon are contributions from the coexisting M-edge bands, which confirms our prediction that they do not affect the topological superconductor on the X edge. It is well established that in a 1D topological superconductor the Majorana fermions appear as end states in real space $[5,6]$. We confirm this by plotting the particle component of the zero-energy mode wave function in Fig. 3(d), where the ribbon's size is $50 \mathrm{~nm}$ in length and $2.5 \mathrm{~nm}$ in width.

\section{EFFECT OF DISORDER}

It is important that Majorana fermions can sustain a certain amount of disorders since in realistic experimental conditions disorders are unavoidable. To explore the disorder effect in this system, we add random on-site potential

$$
\mathcal{H}_{\text {dis }}=\sum_{i, l \alpha} \varepsilon_{i} c_{i, l \alpha}^{\dagger} c_{i, l \alpha},
$$

to the tight-binding model, where $\varepsilon_{i}$ are normally distributed in the range $[-W / 2, W / 2]$. We have simulated two kinds of disorders: (1) disorder covers both bulk and edge, (2) disorder only covers the bulk but not the edge. As shown in Figs. 4(a)4(c), the zero-energy modes as well as the excitation gap are
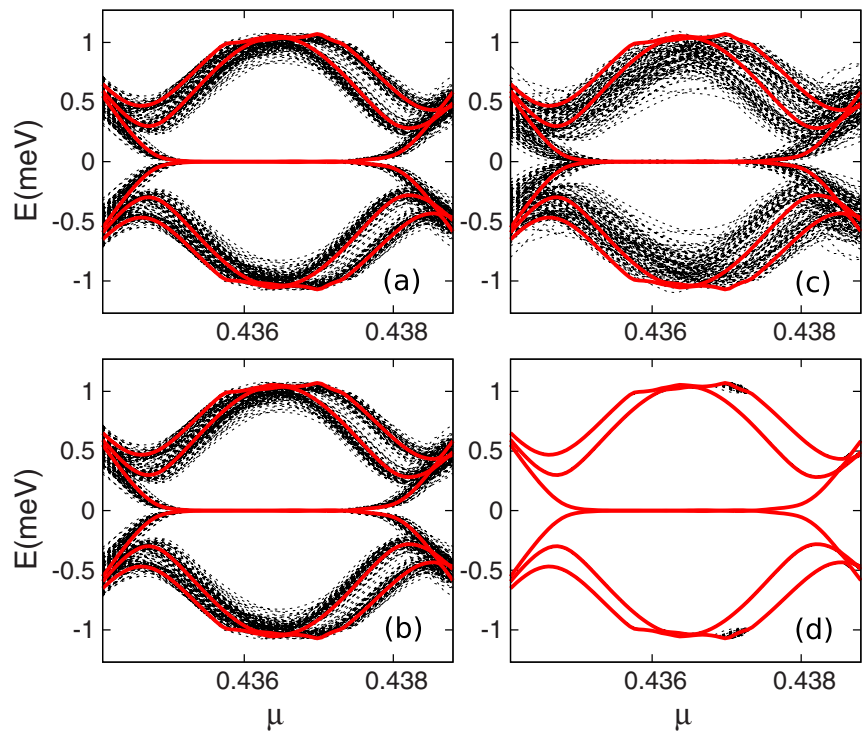

FIG. 4. (Color online) Effect of edge and bulk disorders. In each panel 50 random disorder configurations are collected and the six lowest energy modes for each disorder configuration are plotted. Red and black curves represent the clean and disordered case, respectively. (a)-(c) Disorders are put on both the ribbon's bulk and edge. (a) $W=5 \mathrm{meV}$; (b) $W=10 \mathrm{meV}$; (c) $W=20 \mathrm{meV}$; (d) disorders are put only on the ribbon's bulk but not on the edge, $W=200 \mathrm{meV}$. $\Delta=1 \mathrm{mev}, V_{z}=2 \mathrm{meV}, L / a=400$.

robust against edge disorders up to $W \sim 10 \Delta(W / \Delta$ can be much larger for a smaller $\Delta$ ). For even stronger edge disorders the excitation gap starts to diminish and the zero-energy modes gain splitting. An important advantage of this proposed system is that the topological superconductor resides only on the edge; as a result it gains strong immunity from bulk disorders. This is demonstrated in Fig. 4(d) where the zero-energy modes and excitation gap remain totally intact despite the strong disorder in the bulk.

\section{MATERIAL CONSIDERATIONS}

In the DFT band structures of $\mathrm{MX}_{2}$ zigzag ribbon [Figs. 1(b) and 2(c)], three pairs of in-gap edge states exist connecting the two inequivalent valleys. All of them consist dominantly of the $d$ orbitals from the $\mathrm{M}$ atoms of the ribbon's outermost zigzag chain. Notably two of these edge states have parabolic dispersions at their band minimum/maximum near $k a=\pi$, which suits our purpose to find an effective Hamiltonian like Eq. (3). The two parabolic bands are also fully captured in the tight-binding model. The edge state phenomenon is similar in all $\mathrm{MX}_{2}$ zigzag ribbons we have calculated. After including SOC, the Rashba-type SOC in the edge band is evident when zooming in the band bottom at $k a=\pi$ [Fig. 2(c)]. We have also calculated ribbons with different width. We find these well localized edge states start to exist in very narrow ribbons $\left(N_{c}=4\right)$.

Because the zigzag edges are generally more stable than the armchair edges, during nanoribbon synthesis the zigzag nanoribbons dominate [44-46]. In particular, the X-edge shows maximal stability among all the edge configurations 
of monolayer $\mathrm{MX}_{2}[44,47,48]$. On the $\mathrm{M}$ edge the $\mathrm{M}$ atoms are directly exposed and prone to imperfections. Hence here we emphasize the usage of the $\mathrm{X}$ edge for this theoretical proposal. Missing $\mathrm{X}$ atoms on the $\mathrm{X}$ edge will not be fatal to the edge modes there, unless the $M$ atoms on the $\mathrm{X}$ edge also show defects. In the experimentally grown $\mathrm{MoS}_{2}$ and $\mathrm{WS}_{2}$ nanoribbon, the $\mathrm{S}$ edge was found to be perfect without defects $[44,46]$. Furthermore, edge states have been observed in $\mathrm{MoS}_{2}$ nanoflakes using STM $[40,41]$.

The Fermi surface in DFT calculations of suspended $\mathrm{MX}_{2}$ is typically in the band gap and close to the valence band top. However, both $n$-type [34,51,52] and $p$-type [53,54] conductivities have been reported in transport measurements, suggesting a wide-range tunability for the chemical potentials [55]. The material synthesis and device fabrication of monolayer $\mathrm{MX}_{2}$ are rapidly developing because of their potential applications in the next generation of electronics. The nanoribbon samples for a possible experimental realization for our proposed setup have become readily available [44-46]. The monolayer's 2D nature and similarity with graphene also make many well-developed 2D device engineering and fabrication techniques directly applicable.

Currently there is no experimental measurement of $g$ factors in monolayer $\mathrm{MX}_{2}$. However, the Zeeman field $V_{z}$ only need be larger than the proximity induced superconducting order parameter, which may be at the order of $0.05 \mathrm{meV}$ in realistic experiments [10]. For a typical $g$-factor 2 , it corresponds to a magnetic field of $1 \mathrm{~T}$. It should also be possible to proximity induce $s$-wave $\mathrm{NbSe}_{2}$ superconductor thin film on TMDs since it has the same lattice structure as $\mathrm{MX}_{2}$. The atomically thin layer of $\mathrm{MX}_{2}$ is also a big advantage when considering the superconducting coherence length. Although the SOC energy is very large in this system, the experiment temperature scale would mainly be limited by the excitation gap, which is $1 \sim 10 \mathrm{~K}$ depending on the Zeeman energy $V_{z}$ and proximity induced pairing potential $\Delta$ as shown in Fig. 3 .

\section{CONCLUSION}

The advantage of the proposed platform can be summarized in such a few aspects: (1) The single edge band is well isolated in the middle of a relatively large bulk band gap, which would lead to a minimal background signal in the zero-bias peak measurements for detecting Majorana fermions. The well known multiband problem in the semiconductor nanowires is successfully avoided [21]. More importantly, the platform is a true 1D system localizing on a single atomic chain resulting in strong immunity from the bulk's disorders as we have demonstrated. (2) The system is atomically thick, which would lead to robust and uniform superconducting proximity effect when placed on top of the $s$-wave superconductor. It would also result in efficient gate tunability, which has already been demonstrated in transport measurements with the monolayer [34,56]. (3) The large effective mass and large effective Rashba SOC in this platform is unparalleled to the conventional semiconductor nanowires. As a result, the proximity induced superconducting pairing and the associated Majorana fermions can be robust against disorders.

\section{ACKNOWLEDGMENTS}

R.C. and C.Z. are supported by ARO (W911NF12-1-0334), AFOSR (FA9550-13-1-0045), and NSF-PHY (1104546). G.B.L. acknowledges supports by the NFSC (Grant No. 11304014), the 973 Program (Grant No. 2013CB934500) of China, and the BIT Basic Research Funds (Grants No. 20131842001 and No. 20121842003). W.Y. acknowledges support by HKSAR Research Grant Council (HKU705513P), and Croucher Foundation under the Croucher Innovation Award. X.X is supported by U.S. DOE, BES, Division of Materials Sciences and Engineering (DE-SC0008145). D.X. acknowledges support by the U.S. Department of Energy, Office of Basic Energy Sciences, Materials Sciences and Engineering Division, and AFOSR (FA9550-12-1-0479). The authors acknowledge the Texas Advanced Computing Center (TACC) at The University of Texas at Austin for providing HPC resources that have contributed to the research results reported within this paper.

\section{APPENDIX}

Here we elaborate on how the BdG calculation for Fig. 3 is implemented on the zigzag ribbon's lattice. Denote the spinindependent $3 \times 3$ hopping matrix from the nearest $\mathrm{M}$ atoms at site $i$ to $j$ as $T_{i j}$ and the on-site potential matrices as $H_{o n}=$ $\operatorname{diag}\left(\epsilon_{1} \epsilon_{2} \epsilon_{2}\right)$ [42]. Then the real-space $\mathrm{BdG}$ equation can be written as

$$
\begin{gathered}
\sum_{j}\left(\begin{array}{cccc}
H_{\uparrow \uparrow} & 0 & 0 & \Delta^{\prime} \\
0 & H_{\downarrow \downarrow} & -\Delta^{\prime} & 0 \\
0 & -\Delta^{\prime *} & -H_{\uparrow \uparrow}^{*} & 0 \\
\Delta^{\prime *} & 0 & 0 & -H_{\downarrow \downarrow}^{*}
\end{array}\right)_{i j}\left(\begin{array}{c}
u_{n j}^{\uparrow} \\
u_{n j}^{\downarrow} \\
v_{n j}^{\uparrow} \\
v_{n j}^{\downarrow}
\end{array}\right) \\
=\varepsilon_{n}\left(\begin{array}{c}
u_{n i}^{\uparrow} \\
u_{n i}^{\downarrow} \\
v_{n i}^{\uparrow} \\
v_{n i}^{\downarrow}
\end{array}\right),
\end{gathered}
$$

where $\sigma \equiv\{\uparrow, \downarrow\}, H_{\sigma \sigma, i j}=T_{i j}$ when $i \neq j$, and $H_{\sigma \sigma, i i}=$ $H_{o n} \pm \frac{\lambda}{4} L_{z}, \Delta^{\prime}=\delta_{i j} \cdot I_{3} \otimes \Delta . u_{n i}^{\sigma}$ and $v_{n i}^{\sigma}$, each being a $3 \times$ 1 vector, are the components of the $n$th quasiparticle wave function at site $i ; \varepsilon_{n}$ is the corresponding energy eigenvalue. The low energy spectrum and wave function is then obtained by using the sparse matrix eigensolver in MATLAB.

Our first-principle calculations are performed using the allelectron full-potential linearized augmented-planewave (FPLAPW) method [57]. The SOC is included in terms of the second-variational method with scalar-relativistic orbitals as a basis. LDA exchange-correlation functionals were used. The ribbon lattices were fully relaxed in VASP [58] package until the force on each atom was less than $0.01 \mathrm{eV} / \AA$. The resulting edge structures have slightly contracted $\mathrm{M}-\mathrm{X}$ bonds compared with the bulk [47], further breaking the lattice symmetries at the edge. The energy cutoff of plane wave basis was set to $600 \mathrm{eV}$. Vacuum space between layers was greater than $16 \AA$. Construction of our tight-binding model is described in detail in Ref. [42]. 
[1] F. Wilczek, Nat. Phys. 5, 614 (2009).

[2] C. Nayak, S. H. Simon, A. Stern, M. Freedman, and S. Das Sarma, Rev. Mod. Phys. 80, 1083 (2008).

[3] L. Fu and C. L. Kane, Phys. Rev. Lett. 100, 096407 (2008).

[4] C. Zhang, S. Tewari, R. M. Lutchyn, and S. Das Sarma, Phys. Rev. Lett. 101, 160401 (2008).

[5] J. D. Sau, R. M. Lutchyn, S. Tewari, and S. Das Sarma, Phys. Rev. Lett. 104, 040502 (2010).

[6] R. M. Lutchyn, J. D. Sau, and S. Das Sarma, Phys. Rev. Lett. 105, 077001 (2010).

[7] Y. Oreg, G. Refael, and F. von Oppen, Phys. Rev. Lett. 105, 177002 (2010).

[8] J. Alicea, Phys. Rev. B 81, 125318 (2010).

[9] L. Mao, M. Gong, E. Dumitrescu, S. Tewari, and C. Zhang, Phys. Rev. Lett. 108, 177001 (2012).

[10] V. Mourik, K. Zuo, S. M. Frolov, S. R. Plissard, E. P. A. M. Bakkers, and L. P. Kouwenhoven, Science 336, 1003 (2012).

[11] M. T. Deng, C. L. Yu, G. Y. Huang, M. Larsson, P. Caroff, and H. Q. Xu, Nano Lett. 12, 6414 (2012).

[12] A. Das, Y. Ronen, Y. Most, Y. Oreg, M. Heiblum, and H. Shtrikman, Nat. Phys. 8, 887 (2012).

[13] L. P. Rokhinson, X. Liu, and J. K. Furdyna, Nat. Phys. 8, 795 (2012).

[14] J. Liu, A. C. Potter, K. T. Law, and P. A. Lee, Phys. Rev. Lett. 109, 267002 (2012).

[15] D. Bagrets and A. Altland, Phys. Rev. Lett. 109, 227005 (2012).

[16] F. Pientka, G. Kells, A. Romito, P. W. Brouwer, and F. von Oppen, Phys. Rev. Lett. 109, 227006 (2012).

[17] E. J. H. Lee, X. Jiang, R. Aguado, G. Katsaros, C. M. Lieber, and S. De Franceschi, Phys. Rev. Lett. 109, 186802 (2012).

[18] D. I. Pikulin, J. P. Dahlhaus, M. Wimmer, H. Schomerus, and C. W. J. Beenakker, New J. Phys. 14, 125011 (2012).

[19] S. Das Sarma, J. D. Sau, and T. D. Stanescu, Phys. Rev. B 86, 220506 (2012).

[20] M. Wimmer, A. R. Akhmerov, M. V. Medvedyeva, J. Tworzydlo, and C. W. J. Beenakker, Phys. Rev. Lett. 105, 046803 (2010).

[21] A. C. Potter and P. A. Lee, Phys. Rev. Lett. 105, 227003 (2010).

[22] R. M. Lutchyn, T. D. Stanescu, and S. Das Sarma, Phys. Rev. Lett. 106, 127001 (2011).

[23] T. D. Stanescu, R. M. Lutchyn, and S. Das Sarma, Phys. Rev. B 84, 144522 (2011).

[24] A. C. Potter and P. A. Lee, Phys. Rev. B 83, 184520 (2011).

[25] J. Alicea, Y. Oreg, G. Refael, F. V. Oppen, and M. P. A. Fisher, Nat. Phys. 7, 412 (2011).

[26] F. Fu and C. L. Kane, Phys. Rev. B 79, 161408(R) (2009).

[27] I. Knez, R.-R. Du, and G. Sullivan, Phys. Rev. Lett. 109, 186603 (2012).

[28] X.-L. Qi and S.-C. Zhang, Rev. Mod. Phys. 83, 1057 (2011).

[29] A. C. Potter and P. A. Lee, Phys. Rev. B 85, 094516 (2012).

[30] J. D. Sau, S. Tewari, and S. Das Sarma, Phys. Rev. B 85, 064512 (2012).

[31] S. Tewari and J. D. Sau, Phys. Rev. Lett. 109, 150408 (2012).

[32] D. Xiao, G.-B. Liu, W. Feng, X. Xu, and W. Yao, Phys. Rev. Lett. 108, 196802 (2012).
[33] K. F. Mak, C. Lee, J. Hone, J. Shan, and T. F. Heinz, Phys. Rev. Lett. 105, 136805 (2010).

[34] B. Radisavljevic, A. Radenovic, J. Brivio, V. Giacometti, and A. Kis, Nat. Nanotech. 6, 147 (2011).

[35] T. Cao, F. Feng, J. Shi, Q. Niu, and E. Wang, Nat. Commun. 3, 887 (2012).

[36] K. F. Mak, K. He, J. Shan, and T. F. Heinz, Nat. Nanotech. 7, 494 (2012).

[37] H. Zeng, J. Dai, W. Yao, D. Xiao, and X. Cui, Nat. Nanotech. 7, 490 (2012).

[38] S. Wu, J. S. Ross, G. Liu, G. Aivazian, A. Jones, Z. Fei, W. Zhu, D. Xiao, W. Yao, D. Cobden, and X. Xu, Nat. Phys. 9, 149 (2013).

[39] J. S. Ross, S. Wu, H. Yu, N. J. Ghimire, A. M. Jones, G. Aivazian, J. Yan, D. G. Mandrus, D. Xiao, W. Yao, and X. Xu, Nat. Commun. 4, 1474 (2013).

[40] S. Helveg, J. V. Lauritsen, E. Laegsgaard, I. Stensgaard, J. K. Nørskov, B. S. Clausen, H. Topsøe, and F. Besenbacher, Phys. Rev. Lett. 84, 951 (2000).

[41] M. V. Bollinger, J. V. Lauritsen, K. W. Jacobsen, J. K. Nørskov, S. Helveg, and F. Besenbacher, Phys. Rev. Lett. 87, 196803 (2001).

[42] G.-B. Liu, W.-Y. Shan, Y. Yao, W. Yao, and D. Xiao, Phys. Rev. B 88, 085433 (2013).

[43] Z. Y. Zhu, Y. C. Cheng, and U. Schwingenschlogl, Phys. Rev. B 84, 153402 (2011).

[44] Z. Wang, H. Li, Z. Liu, Z. Shi, J. Lu, K. Suenaga, S. Joung, T. Okazaki, Z. Gu, J. Zhou, Z. Gao, G. Li, S. Sanvito, E. Wang, and S. Iijima, J. Am. Chem. Soc. 132, 13840 (2010).

[45] Z. Wang, K. Zhao, H. Li, Z. Liu, Z. Shi, J. Lu, K. Suenaga, S. Joung, T. Okazaki, Z. Jin, Z. Gu, Z. Gaob, and S. Iijima, J. Mater. Chem. 21, 171180 (2011).

[46] Z. Liu, K. Suenaga, Z. Wang, Z. Shi, E. Okunishi, and S. Iijima, Nat. Commun. 2, 213 (2011).

[47] Y. Li, Z. Zhou, S. Zhang, and Z. Chen, J. Am. Chem. Soc. 130, 16739 (2008).

[48] H. Pan and Y. Zhang, J. Mater. Chem. 22, 7280 (2012).

[49] E. Erdogan, I. H. Popov, A. N. Enyashin, and G. Seifert, Eur. Phys. J. B 85, 33 (2012).

[50] T. D. Stanescu, R. M. Lutchyn, and S. Das Sarma, Phys. Rev. B 87, 094518 (2013).

[51] S. Wu, C. Huang, G. Aivazian, J. S. Ross, D. H. Cobden, and X. Xu, ACS Nano. 7, 2768 (2013).

[52] A. M. van der Zande, P. Y. Huang, D. A. Chenet, T. C. Berkelbach, Y. You, G. Lee, T. F. Heinz, D. R. Reichman, D. A. Muller, and J. C. Hone, Nat. Mat. 12, 554 (2013).

[53] Z. Zeng, Z. Yin, X. Huang, H. Li, Q. He, G. Lu, F. Boey, and H. Zhang, Angew. Chem. Int. Ed. 50, 11093 (2011).

[54] Y. Zhan, Z. Liu, S. Najmaei, P. M. Ajayan, and J. Lou, Small 8, 966 (2012).

[55] K. Dolui, I. Rungger, and S. Sanvito, Phys. Rev. B 87, 165402 (2013).

[56] Y. Zhang, J. Ye, Y. Matsuhashi, and Y. Iwasa, Nano Lett. 12, 1136 (2012).

[57] http://elk.sourceforge.net

[58] G. Kresse and J. Furthmuller, Phys. Rev. B 54, 11169 (1996). 